\title{
Assessment of Functional Connectome in End-Stage Organ Disease Patients after Life-Threatening Surgery
}

\author{
G. Sparacia ${ }^{1}$, G. Parla ${ }^{1}$, M. Shahriari $^{2}$, V. Lo Re ${ }^{1}$, G. Mamone ${ }^{1}$, A. Iaia ${ }^{2}$, R. Miraglia ${ }^{1}$

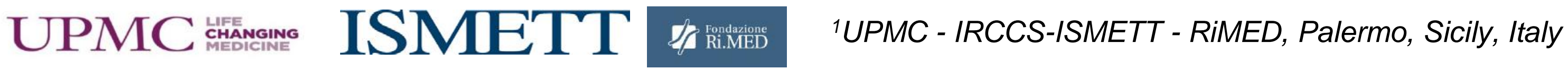 \\ ChristianaCare- ${ }^{2}$ ChristianaCare Health System, Newark, DE, USA
}

All Authors have no financial conflicts or relevant disclosures

\section{Purpose}

The purpose of this study was to assess the organization of intrinsic functional brain networks (functional connectome) in neurologically asymptomatic patients with end-stage organ disease who had undergone major surgery for life-threating conditions and compare it to a control group.

\section{Materials and Methods}

Resting-state functional magnetic resonance imaging (rest-fMRI) was performed in 10 adult, post-operative patients with endstage organ disease. The patients ( 7 men, 3 women, mean age $57.9 \pm 7.4$ years) had undergone: $n=4$ coronary artery bypass graft for heart failure, $n=2$ orthotopic liver transplantation for nonalcoholic cirrhotic liver failure, $n=3$ mitral valve repair for heart failure, $n=1$ pancreaticoduodenectomy for pancreatic papillary tumor. Rest-fMRI was acquired within 48 hours after intensive care unit discharge. Structural brain MR imaging was acquired with T1-weighted, T2-weighted, diffusion weighted imaging, and susceptibility weighted imaging along with 3D isotropic T1weighted MR images. MR examinations were performed on a $3 \mathrm{~T}$ MR scanner (Discovery 750w, General Electric Healthcare, Milwaukee, WI, USA). Ten age- and sex-matched healthy controls were studied with the same protocol. Brain functional networks were analyzed by calculating the interregional correlation of low-frequency fluctuations in spontaneous brain activity. Weighted graph-based models were employed to topologically recognize reproducibly determined large-scale functional networks, including default mode, salience, dorsal attention, sensorimotor, visual and language networks using a seed-based approach with Functional Connectivity Toolbox (CONN) (https://web.conn-toolbox.org/) running under MatLab (The MathWorks Inc., Natick, MA, USA).

A $p$-uncorrected $<0.0025$ was used to threshold connectomes for voxel-wise paired statistical analysis to take in account the lateralization effect. Network comparisons were thresholded using a False Discovery Rate (FDR) cluster-level correction approach in patients and controls using the MatLab function "mafdr" for $p$-uncorrected $<0.0025$ obtaining the $p$-FDR $<0.077$.

\section{Results}

Functional connectivity in patients and controls was successfully assessed for the default mode, salience, dorsal attention, sensorimotor, visual, and language networks of the brain. There was statistically significant $(p<0.05)$ reduced connectivity between seeds in the default mode, salience, sensorimotor, and language networks in patients compared to controls (Fig. 1).

No acute structural lesions were observed in patients at MR imaging.

\section{Conclusion}

Assessment of functional connectome in end-stage organ disease patients is feasible in routine clinical setting. Rest-fMRI can demonstrate reduced connectivity of several intrinsic functional networks in critically ill, post-operative patients and it may be a useful prognostic indicator of early and long-term clinical outcome.

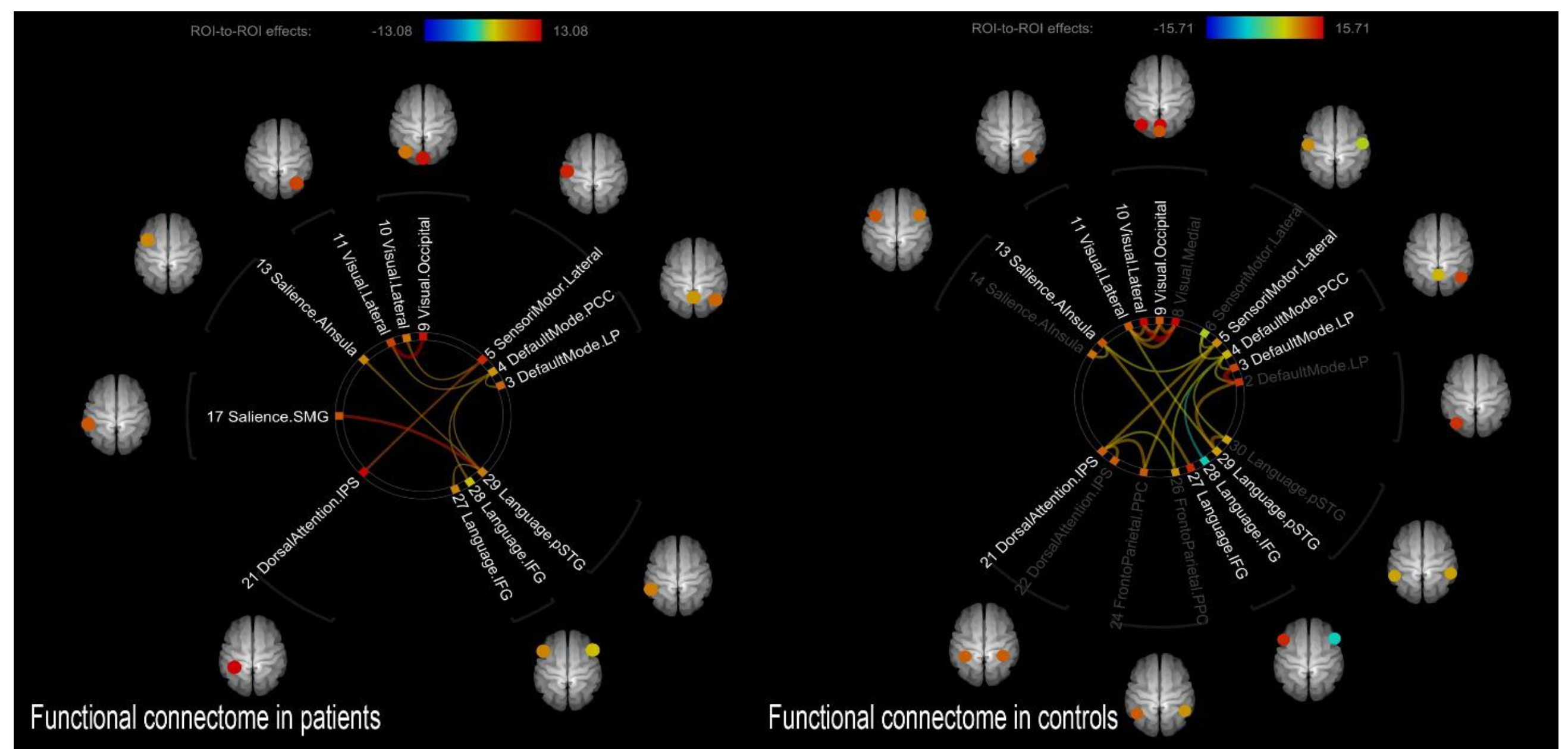

References

$\mathrm{Ma} X$, et al. Aberrant functional connectome in neurologically asymptomatic patients with end-stage renal disease. Plos One 2015;10:e0121085.

Lariviere $\mathrm{S}$, et al. Disrupted functional network integrity and flexibility after stroke: Relation to motor impairments. Neurolmage. Clinical 2018;19:883-891.

Cheng $Y$, et al. Longitudinal Intrinsic Brain Activity Changes in Cirrhotic Patients before and One Month after Liver Transplantation. Korean Journal of Radiology, 2017;18,370377.

Zhang XD, et al. Long-and short-range functional connectivity density alteration in non-alcoholic cirrhotic patients one month after liver transplantation: A resting-state fMRI study. Brain Research, 2015;1620:177187.

Whitfield-Gabrieli S, Nieto-Castanon A Conn: a functional connectivity toolbox for correlated and anticorrelated brain networks. Brain Connect. 2012; 2:125-141. 